\title{
Within- and between-channel gap detection in the human auditory cortex
}

\section{Document Version}

Final published version

Link to publication record in Manchester Research Explorer

\section{Citation for published version (APA):}

Heinrich, A., Alain, C., \& Schneider, B. A. (2004). Within- and between-channel gap detection in the human auditory cortex. NeuroReport, 15(13), 2051-2056.

\section{Published in:}

NeuroReport

\section{Citing this paper}

Please note that where the full-text provided on Manchester Research Explorer is the Author Accepted Manuscript or Proof version this may differ from the final Published version. If citing, it is advised that you check and use the publisher's definitive version.

\section{General rights}

Copyright and moral rights for the publications made accessible in the Research Explorer are retained by the authors and/or other copyright owners and it is a condition of accessing publications that users recognise and abide by the legal requirements associated with these rights.

\section{Takedown policy}

If you believe that this document breaches copyright please refer to the University of Manchester's Takedown Procedures [http://man.ac.uk/04Y6Bo] or contact uml.scholarlycommunications@manchester.ac.uk providing relevant details, so we can investigate your claim.

\section{OPEN ACCESS}




\title{
Within- and between-channel gap detection in the human auditory cortex
}

\author{
Antje Heinrich, ${ }^{1, C A}$ Claude Alain ${ }^{1,2}$ and Bruce A. Schneider' \\ 'Department of Psychology, University of Toronto at Mississauga, 3359 Mississauga Rd, Mississauga, ON, L5L IC6; ${ }^{2}$ Rotman Research Institute, \\ Baycrest Centre for Geriatric Care \& Department of Psychology, University of Toronto \\ CACorresponding Author: aheinric@utm.utoronto.ca. \\ Received I3 May 2004; accepted 2I June 2004
}

\begin{abstract}
We examined the neural correlates associated with a short gap between two identical pure tones (within-channel) and between two different tones (between-channel) in an odd-ball paradigm. Gap durations were selected such that a gap between identical tones was as discriminable as a gap between two different tones. Spatio-temporal dipole source modeling of electrophysiological data revealed a significant difference between standard and deviant gap stimuli, with mismatch negativity responses that were
\end{abstract}

comparable in amplitude and latency for within- and betweenchannel conditions. Therefore, the ability to automatically register discontinuity (i.e., gap) within and between channels is comparable despite significant differences in gap size. The dipole source modeling suggests that both within- and between-gap signals are represented in or near the primary auditory cortex. NeuroReport 15:205I-2056 (c) 2004 Lippincott Williams \& Wilkins.

Keywords: Auditory; Event-related potential (ERP); Gap detection; Mismatch negativity (MMN); Temporal resolution

\section{INTRODUCTION}

Numerous studies have shown that gap detection thresholds are sensitive to the spectral similarities of the markers delimiting the gap. For example, markers of identical frequency allow for greater temporal acuity than markers of dissimilar frequency [1-4]. Phillips and colleagues propose that spectrally dissimilar sounds activate different peripheral auditory neurons, which results in the activation of different perceptual channels [5,6]. Applying this account to pure tone gap detection tasks, markers that differ in frequency are assumed to stimulate different perceptual channels. In this case, the gap detection task is referred to as between-channel. Markers of identical frequency stimulate the same auditory channels and the task is referred to as within-channel.

The perceptual operations that support gap detection are likely to differ for within- and between-channel gap detection tasks. If the representational overlap between activated channels is substantial because the two markers stimulate the same or similar channels, the gap can be detected as a discontinuity between co-activated neural representations. On the other hand, if there is insufficient overlap between activated representations, gap detection has to rely on the relative timing of activity between the offset of the neural activity pattern of the leading marker and the onset of the pattern representing the trailing marker. Phillips et al. suggest that the central computation associated with relative time-stamping between channels has an inherently poorer temporal acuity, which results in higher gap detection thresholds $[7,8]$.

The present study aims to investigate the hypothesis of dissimilar auditory mechanisms in within- and between- channel processing using the mismatch negativity (MMN) wave. Infrequent deviants occurring in a sequence of standard stimuli typically elicit the MMN wave. Previous studies have shown that more salient stimuli with longer gaps give rise to higher amplitudes and shorter latencies [9-13]. In those studies, both gap duration and perceptual accuracy were confounded, making it difficult to determine whether the increase in MMN amplitude with gap size reflected gap duration per se or perceptual saliency. In addition, no study, to our knowledge, has compared MMN responses elicited by within- and between-channel gap stimuli. In the present study we controlled for perceptual saliency by presenting participants with within- and between-channel gap stimuli that led to identical perceptual accuracy. A previous study suggested that energy differences between the standard and comparison stimulus may contribute to gap detection thresholds [14]. Hence, in the present experiment we also equated for stimulus energy. For both within- and between-channel conditions, gaps were selected that were equally discriminable.

\section{MATERIALS AND METHODS}

Participants: Twelve young adults (mean ( \pm s.d.) age: $20.75 \pm 1.8$ years; seven females) with normal hearing $(\leq 15 \mathrm{~dB}$ HL at the $1 \mathrm{kHz}$ and $2 \mathrm{kHz}$ test frequencies in the left ear) constituted the final sample of the study. Two volunteers withdrew after behavioral testing due to personal reasons.

Stimuli: The stimuli were digitally generated with a sampling rate of $20 \mathrm{kHz}$ and converted to analog form 
using a 16-bit Tucker Davis Technology digital-to-analog converter. To obtain the stimuli, amplitude envelopes for gap and no-gap sounds were constructed by summing a series of Gaussian functions (s.d. $1 \mathrm{~ms}$ ), spaced $1 \mathrm{~ms}$ apart, following the procedure described by Schneider and Hamstra [15]. Figure 1 (top) displays the amplitude envelopes (solid line) for two $20 \mathrm{~ms}$ markers separated by a $20 \mathrm{~ms}$ gap. The duration of each marker is specified by the $1 / 2$ power points of the marker envelope. The duration of each gap is specified by the difference in time between the $1 / 2$ power point at the end of the first marker envelope and the equivalent point at the beginning of the second marker envelope. Note that the amplitude envelope decays to zero during the gap for long gap durations. However, for very small gap durations, the amplitude envelope does not decay to zero and the sum of the two markers begins to affect the half power points at the end of the first marker and the beginning of the second marker. To avoid this problem when analyzing data, we used the area between zero and the dotted line to index gap duration. For gap durations greater than $2 \mathrm{~ms}$, this area is directly proportional to the gap between the $1 / 2$ power points from the end of the first marker to the beginning of the second marker. All analyses were conducted on the area under this dotted line and translated back into gap durations using this linear relationship.

The middle panel plots the envelopes of the markers for a no-gap comparison stimulus whose total duration equals that of the gap stimulus. Note that the shape of each marker is the same but that they overlap to such an extent that the sum of the two envelopes (indicated by the dotted line) is flat between the beginning of the first marker and the end of the second marker so that there is no break in sound energy.

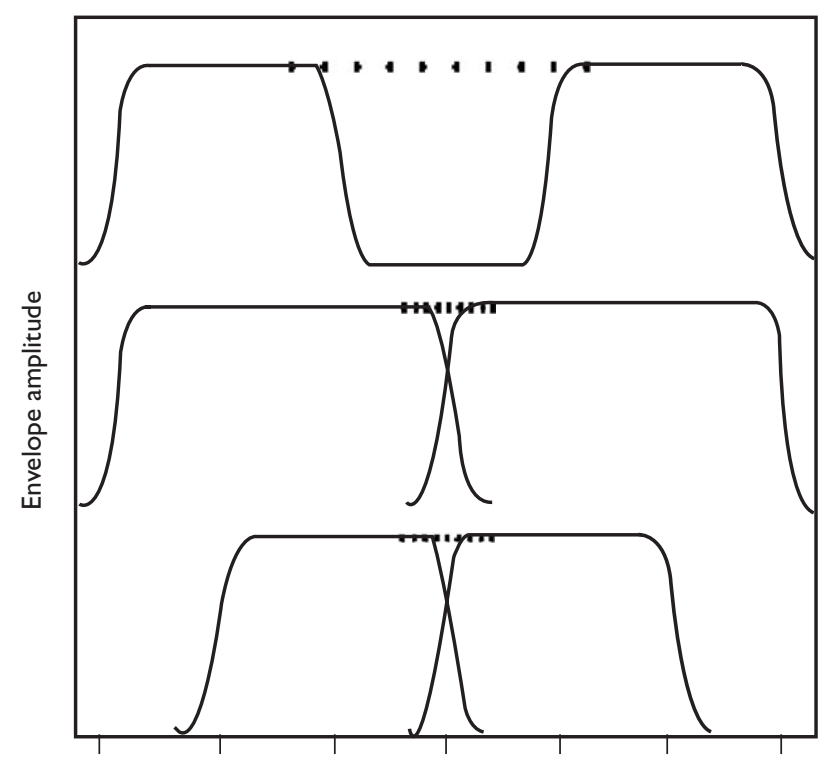

Time in 10 ms steps

Fig. I. Top: The envelopes of two $20 \mathrm{~ms}$ markers separated by a $20 \mathrm{~ms}$ gap. The area between the dotted line and the sum of the two envelopes indexes the extent of the gap. Middle: The envelopes of two overlapping $30 \mathrm{~ms}$ markers. Bottom: The envelopes of two 2 ms markers with overlapping envelopes. Here the duration of each marker in the comparison (no gap) stimulus is the same as in the gap stimulus.
Note also that the duration of the sum of the two envelopes (61 $\mathrm{ms}$ ) approximates the total duration of the gap stimulus (20 ms marker, $20 \mathrm{~ms}$ gap, $20 \mathrm{~ms}$ marker). The comparison stimuli were constructed in such a way that they matched gap stimuli in overall duration for odd gap durations (e.g. $9 \mathrm{~ms}$ ) and exceeded them in overall duration by $1 \mathrm{~ms}$ in cases of even gap sizes (e.g. $8 \mathrm{~ms}$ ).

This comparison stimulus was used in the within-channel (WC) condition, and for the between-channel condition in which the total duration of the no-gap comparison stimulus matched that of the gap stimulus (BCM).

The bottom panel shows a comparison stimulus in the between-channel condition in which the duration of each of the markers is the same as in the gap stimulus (betweenchannel, fixed duration, BCF), but the overlap is such that the sum of the two envelopes is flat (dotted line). Hence, in the BCM condition (middle panel) the gap and comparison stimuli are matched in total duration, while in the BCF condition, the comparison stimulus always has a fixed duration ( $40 \mathrm{~ms})$.

These envelopes were normalized so that the total energy was constant for all stimuli. After being normalized, the leading marker was multiplied by a pure tone of 1 or $2 \mathrm{kHz}$, and the lagging marker by a pure tone of the same frequency (within-channel) or different frequency (between-channel). The stimuli were presented at $70 \mathrm{~dB}$ SPL to the left ear over TDH-49 earphones in a single-wall sound-attenuating booth.

Psychoacoustic procedure: Behavioral gap thresholds were determined for all six stimulus conditions in three separate one-hour sessions. A 6-down, 1-up tracking procedure was used in a 2IFC paradigm to determine the $89.1 \%$ accuracy point on the psychometric function [16]. At the beginning of each run, the gap size was set to its maximum value, $31 \mathrm{~ms}$ in within-channel conditions and $61 \mathrm{~ms}$ in between-channel conditions. In each trial, which was initiated by a button press, a gap and a no-gap stimulus were presented, randomly assigned to one of two $500 \mathrm{~ms}$ stimulus intervals. Each stimulus began $100 \mathrm{~ms}$ into the interval and the two intervals were separated by a $100 \mathrm{~ms}$ period of silence.

Participants indicated which interval contained the gap by pressing one of two buttons. Lights on the button box indicated the beginning and the length of each interval and also provided immediate feedback to the participant about the accuracy of their response. Each run was ended after 12 reversals. The gap detection threshold was defined as the average of the last eight reversals. Participants cycled through all six conditions for a total of four times. Order of presentation was counterbalanced in all conditions. The testing was self-paced, initiated by a button press.

Oddball procedure: Participants were presented with sequences of sounds containing deviant (gap) and standard (no-gap) stimuli. The gap duration corresponded to the $89.1 \%$ accuracy point of the psychometric function as determined behaviorally in the first part of the study. The no-gap or standard stimuli were identical to the continuous stimulus used as the comparison tone for the gap stimulus at the $89.1 \%$ threshold level. The stimulus pattern was constructed in such a way that the deviant gap stimulus occurred in $15 \%$ of all presented sounds, with a minimum interval of three standards between two deviants. 
Participants were presented with a total of 1200 stimuli per condition, 180 of which were deviants. Stimuli were presented with a fixed inter-stimulus interval of $500 \mathrm{~ms}$ at $70 \mathrm{~dB}$ SPL to the left ear using TDH-49 headphones. Presentation order of the six conditions was the same as in the behavioral part. All data were recorded in a single recording session of $\sim 90 \mathrm{~min}$. Participants were instructed to ignore the stimuli (passive listening) and watched a subtitled movie of their choice.

Recording and analyses of auditory evoked potentials: Brain activity was recorded from an array of 64 channels including those of the standard 10-20 placements. Eye movements were recorded with electrodes at the outer canthi and at the superior and inferior orbit. The signals were amplified and bandpass filtered $(0.1$ and $40 \mathrm{~Hz}$; sampling rate of $250 \mathrm{~Hz}$ ) via NeuroScan SynAmps, and then stored for offline analysis. During the recording, all electrodes were referenced to $\mathrm{Cz}$; for data analysis, they were re-referenced to an average reference.

ERP epochs were extracted off-line and included $100 \mathrm{~ms}$ pre-stimulus and $500 \mathrm{~ms}$ post-stimulus recording period. Trials contaminated by excessive peak-to-peak deflections $(100 \mu \mathrm{V})$ at channels not adjacent to the eyes were automatically rejected before averaging. The ERPs were averaged separately for each site, stimulus type and condition. For each individual average, the ocular artifacts were corrected using ocular source components through brain electrical source analysis (BESA) software [17]. To improve signal/noise ratio we collapsed over frequency order for each channel condition reducing the conditions from six to three. ERPs were digitally band-pass filtered between 1 and $15 \mathrm{~Hz}$.

In the present study we compared the effects of stimulus type and conditions on the amplitude of the source waveforms obtained from the left and right hemispheres. While conventional ERP analyses are based on a subset of electrodes, the source waveform analyses take into account the whole electrode array (e.g. 64 electrodes) and reduce it into more manageable number of dimension (e.g. two sources, one in each hemisphere). The spatio-temporal source analyses were performed using the BESA 5.1 software package (MEGIS Software, $\mathrm{GmbH}$, Munich). The fit interval of the BESA model with one regional source in each hemisphere was from 80 to $180 \mathrm{~ms}$ after sound onset and encompassed the N1, P2, and MMN waves. To enhance the signal-to-noise ratio, the fit procedure was based on the group mean ERPs elicited by the standard stimuli from all three conditions. The sources were kept symmetrical. The resulting model was held fixed and used as a spatial filter to derive the source waveforms (one in each hemisphere) of all three gap conditions separately. The ANOVAs were performed on mean amplitude (nAm) over selected latency region with condition, stimulus type, and hemisphere as factors.

\section{RESULTS}

Behavioral results: Table 1 shows that in the withinchannel condition, the gap threshold for the $1000 \mathrm{~Hz}$ condition was smaller than in the $2000 \mathrm{~Hz}$ condition $(\mathrm{t}(11)=4.27, p<0.001)$. A 2 (order of frequency) $\times 2$ (marker condition) ANOVA on the between-channel data indicate a significant main effect of frequency order $(F(1,11)=12.01$, $p<0.005$; i.e. ascending frequency order led to a smaller
Table I. Geometric mean (standard deviation) of gap detection thresholds in milliseconds for all within- and between-channel conditions.

\begin{tabular}{lcccc}
\hline & $\begin{array}{c}\text { Within- } \\
\text { channel } \\
(\text { WC) }\end{array}$ & $\begin{array}{c}\text { Between- } \\
\text { channel } \\
(B C M)\end{array}$ & $\begin{array}{c}\text { Between- } \\
\text { channel } \\
(B C F)\end{array}$ \\
\hline $\begin{array}{l}\text { Leading marker } \\
\text { frequency }\end{array}$ & $\mathrm{I} \mathrm{kHz}$ & $1.13(0.48)$ & $5.98(2.70)$ & $8.67(8.61)$ \\
& $2 \mathrm{kHz}$ & $1.60(0.56)$ & $7.12(3.48)$ & $10.90(6.84)$ \\
\hline
\end{tabular}

threshold) and a main effect of marker condition $(\mathrm{F}(1,11)=5.09, p<0.05$; threshold for $\mathrm{BCM}$ is smaller than $\mathrm{BCF})$, but no interaction between these two factors.

Electrophysiological results: Figure 2 shows the group mean ERP responses elicited by the standard and deviant stimuli in all three conditions. All stimuli elicited a small positive wave (P1), a large negative-positive complex (N1P2) peaking respectively at 110 and $200 \mathrm{~ms}$ post-stimulus at frontocentral scalp sites. Our dipole solution reveals generators of the N1-P2 in the superior temporal gyrus near the primary auditory cortex (Fig. 3). The residual variance was $6.45 \%$, suggesting that this model provides a good fit to the scalp-recorded data. For both standard and deviant stimuli, the source waveforms from the hemisphere contralateral to the sound source were comprised of peaks of activity 110 and $200 \mathrm{~ms}$ after sound onset, which corresponded to the N1 and P2 waves of the scalp-recorded data.

The effect of condition on the MMN amplitude was examined over the $125-175 \mathrm{~ms}$ interval. An ANOVA with 3 conditions (WC, BCM, BCF), 2 stimuli (deviant, standard), and 2 hemispheres revealed significant main effects for stimulus type $(\mathrm{F}(1,11)=19.69, p<0.001)$ and hemisphere $(F(1,11)=23.14, p<0.001)$, but not for experimental condition, or for any of the interactions among these three factors. This result confirmed the presence of a significant mismatch, which was larger in the right than in the left hemisphere, but did not differ across experimental condition.

\section{DISCUSSION}

The behavioral results are consistent with studies showing that gap-detection thresholds for pure tones in withinchannel conditions are often in the single digit millisecond range $[15,18]$. Thresholds were considerably higher for between-channel conditions, a result again also in concordance with other studies [1,2]. Interestingly, the gap thresholds did differ for the two sets of between-channel conditions even when there were no energy differences among stimuli. Hence, duration cues may play a role in between-channel gap detection.

In agreement with earlier studies $[19,20]$, auditory N1-P2 waves were best accounted for by sources in the superior temporal gyrus near the primary auditory cortex. The source strength for the P2 wave was greater in the hemisphere contralateral to the sound source. The comparison of the source waveforms elicited by standard and deviant gap stimuli yielded differences in amplitude that corresponded to the MMN of the scalp-recorded data. 
Within-channel

WC
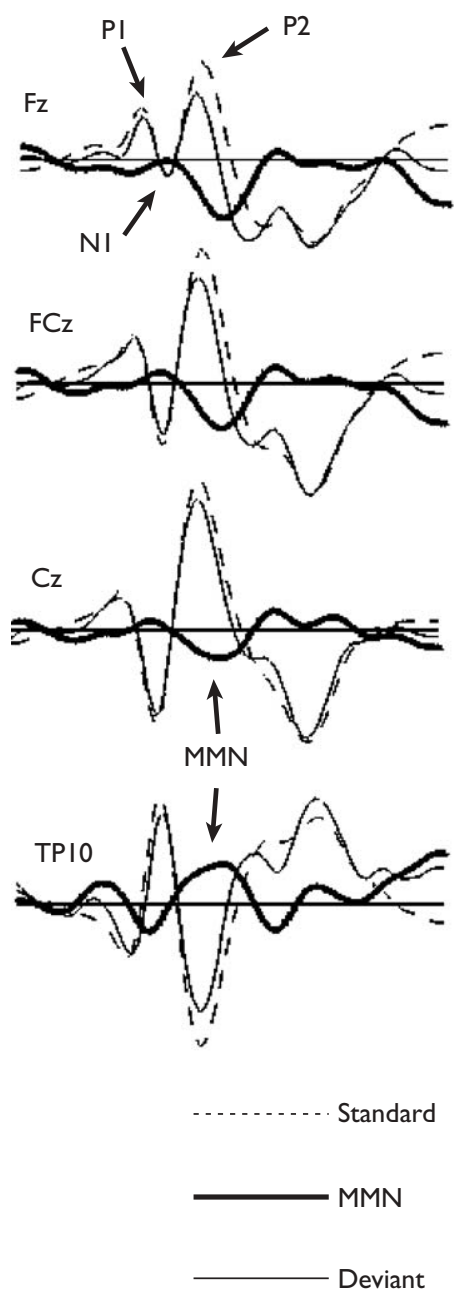

Between-channel

BCM
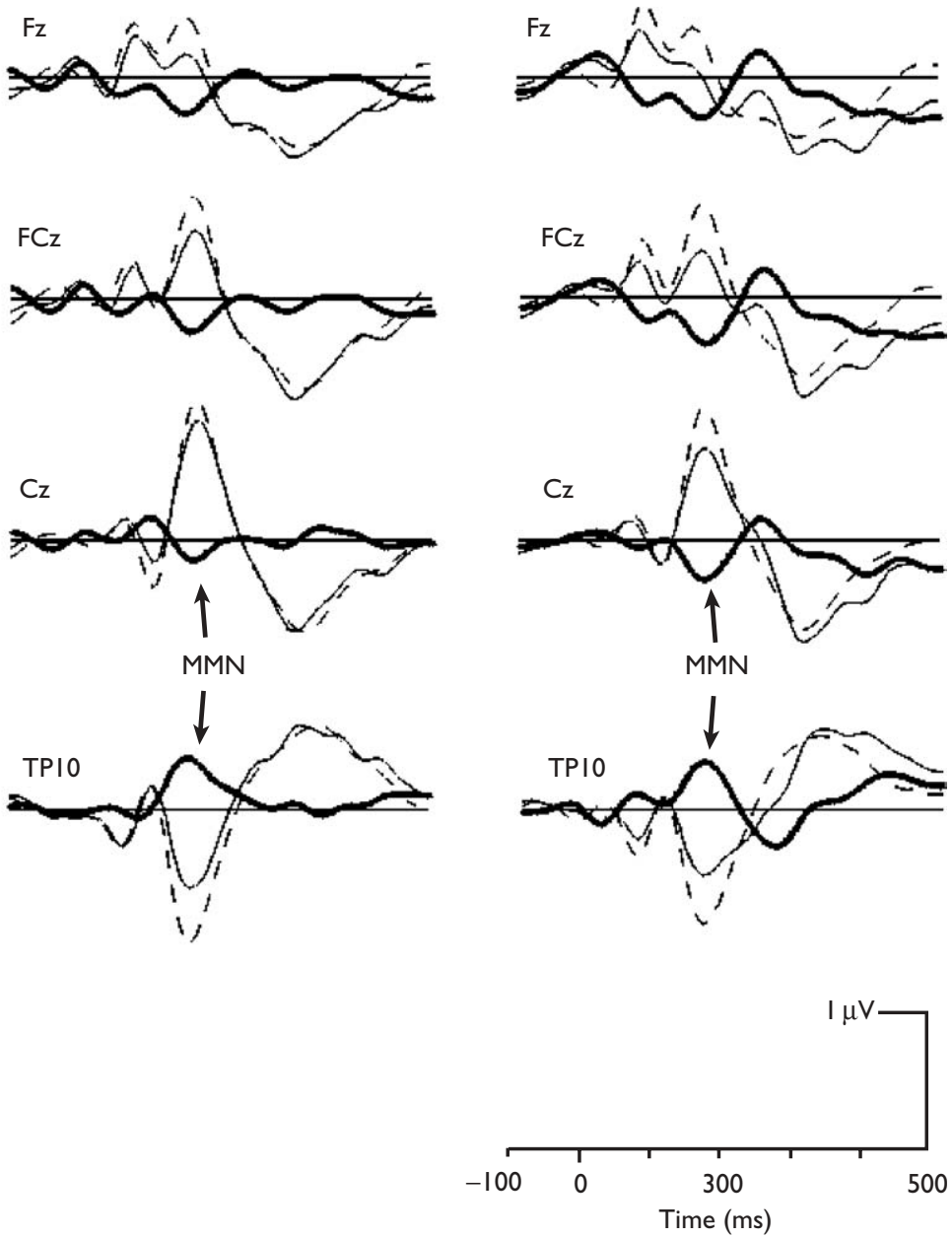

Fig. 2. Group mean event-related potentials for all 12 listeners to deviant (solid line) and standard (dotted line) stimuli for the within- and betweenchannel conditions. Positivity is plotted upward.

In the present study, infrequent discontinuity within or between two information channels generated MMN responses that were comparable in amplitude and latency. This finding replicates and extends those of prior studies [9-11] by showing MMN to both within- and betweenchannel gap signals. More importantly, the amplitude and latency of the MMN was comparable between the conditions despite the fact that the gap for between-channel conditions was about five times larger than that of the within-channel condition and the gap thresholds for the two between-channel condition differed significantly. These findings indicate that MMN amplitude and latency is primarily driven by perceptual saliency (which was comparable among the three conditions) rather than the physical difference between standard and deviant stimuli. This highlights the importance of perceptual saliency when comparing MMN waves for various deviant types in the same or different groups of participants. It has been proposed that the MMN generation depends on sensory memory and a frontotemporal network that includes auditory cortices along the superior temporal gyrus and the dorsolateral prefrontal cortex [21]. Perhaps it should not be surprising that the MMN waves in within- and betweenchannel conditions were comparable given that the MMN wave indexes changes within a general perceptual context, and may be insensitive to specific details in the stimulus (such as whether the leading and lagging markers are identical or differ in frequency).

\section{CONCLUSION}

Behavioral studies suggest different mechanisms for processing within- and between-channel gap signals. In agreement with previous research, we found larger gap thresholds for between- than within-channel gap detection tasks. However, the ability to automatically register equally discriminable within- or between-channel discontinuities generated comparable MMN responses despite significant differences in gap size. This finding indicates that the MMN system operates on the output provided by the mechanisms 
Left

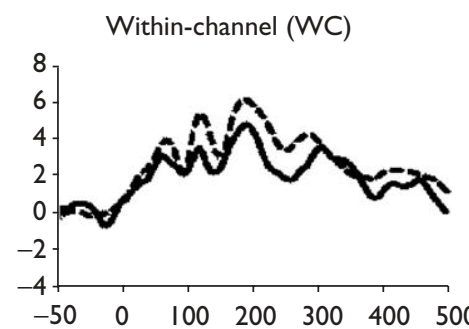

Between-channel (BCM)
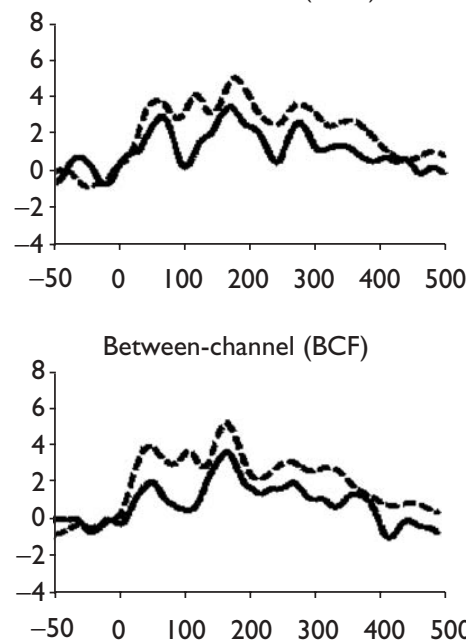

Difference waves

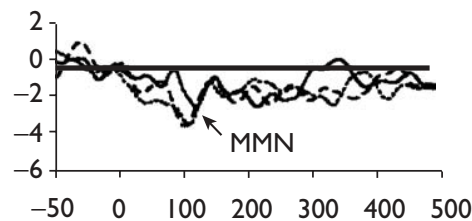

Right
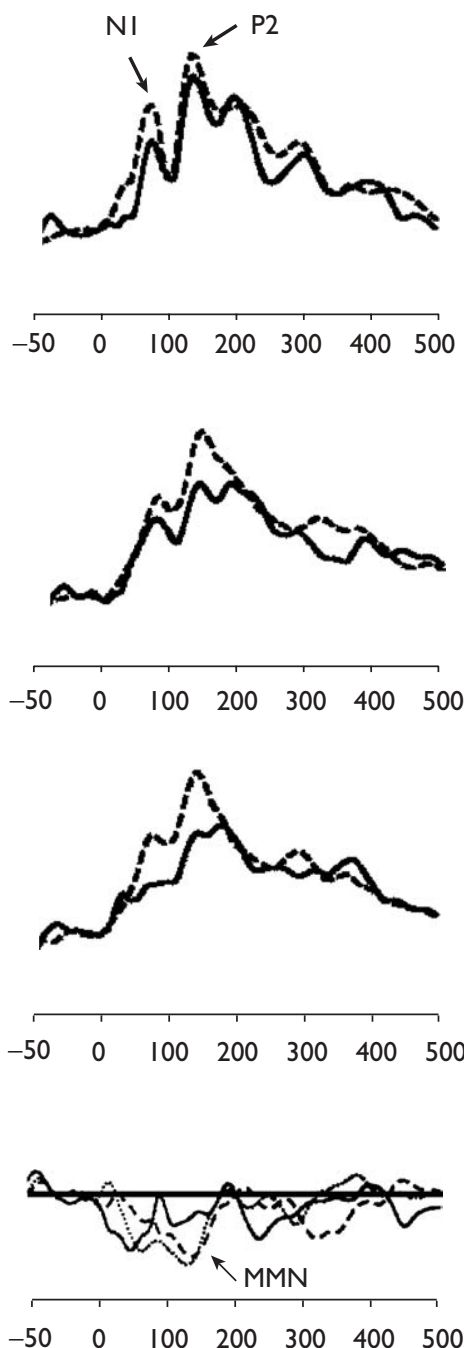

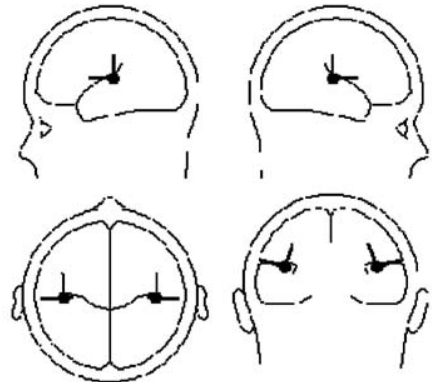

Standard

Deviant
Within-channel

(WC)

Between-channel (BCF)

Between-channel $(\mathrm{BCM})$

Fig. 3. Group mean source waveforms from the left and right hemispheres. The source waveform reflects the combination of activity represented by the tangential, radial and anterior-posterior components of the regional sources.

involved in processing discontinuity within and between channels. Evidence from neuromagnetic recording of middle latency responses shows that the primary auditory cortex can resolve gaps as small as $3 \mathrm{~ms}[22,23]$. Hence, recording of middle-latency evoked responses may be more suitable for investigating the neural underpinning of gap detection. We argue that the primary auditory cortex plays a central role in the computation required for within- and between-channel discontinuity detection and that the MMN wave indexes changes within a more general perceptual context.

\section{REFERENCES}

1. Grose JH, Hall JW III, Buss E and Hatch D. Gap detection for similar and dissimilar gap markers. J Acoust Soc Am 2001; 109:1587-1595.

2. Formby $\mathrm{C}$ and Forrest TG. Detection of silent temporal gaps in sinusoidal markers. J Acoust Soc Am 1991; 89:830-837.

3. Formby C, Gerber MJ, Sherlock LP and Magder LS. Evidence for an across-frequency, between-channel process in asymptotic monaural temporal gap detection. J Acoust Soc Am 1998; 103:3554-3560.
4. Divenyi PL and Danner WF. Discrimination of time intervals marked by brief acoustic pulses of various intensities and spectra. Percept Psychophys 1977; 21:125-142.

5. Phillips DP, Hall SE, Harrington IA and Taylor TL. Central auditory gap detection: a spatial case.J Acoust Soc Am 1998; 103:2064-2068.

6. Phillips DP and Hall SE. Independence of frequency channels in auditory temporal gap detection. J Acoust Soc Am 2000; 108:2957-2963.

7. Phillips DP. Auditory gap detection, perceptual channels, and temporal resolution in speech perception. J Am Acad Audiol 1999; 10: 343-354.

8. Phillips DP, Taylor TL, Hall SE, Carr MM and Mossop JE. Detection of silent intervals between noises activating different perceptual channels: some properties of central auditory gap detection. J Acoust Soc Am 1997; 101:3694-3705.

9. Alain C, McDonald KL, Ostroff JM and Schneider BA. Aging: a switch from automatic to controlled processing of sounds? Psychol Aging 2004; 19:125-133.

10. Bertoli S, Smurzynski J and Probst R. Temporal resolution in young and elderly subjects as measured by mismatch negativity and a psychoacoustic gap detection task. Clin Neurophysiol 2002; 113:396-406.

11. Bertoli S, Heimberg S, Smurzynski J and Probst R. Mismatch negativity and psychoacoustic measures of gap detection in normally hearing subjects. Psychophysiology 2001; 38:334-342. 
12. Desjardins RN, Trainor LJ, Hevenor SJ and Polak CP. Using mismatch negativity to measure auditory temporal resolution thresholds. Neuroreport 1999; 10:2079-2082.

13. Trainor LJ, Samuel SS, Desjardins RN and Sonnadara RR. Measuring temporal resolution in infants using mismatch negativity. Neuroreport 2001; 12:2443-2448.

14. Formby $\mathrm{C}$ and Muir K. Effects of randomizing signal level and duration on temporal gap detection. Audiology 1989; 28:250-257.

15. Schneider BA and Hamstra SJ. Gap detection thresholds as a function of tonal duration for younger and older listeners.J Acoust Soc Am 1999; 106:371-380.

16. Levitt H. Transformed up-down methods in psychoacoustics. J Acoust Soc Am 1971; 49:467-477.

17. Picton TW, Alain C, Otten L, Ritter W and Achim A. The correction of ocular artifacts: a topographic perspective. Clin Neurophysiol 2000; 111:53-65.
18. Moore BCJ, Peters RW and Glasberg BR. Detection of temporal gaps in sinusoids: effects of frequency and level. J Acoust Soc Am 1993; 93:1563-1570.

19. Scherg M, Vajsar J and Picton TW. A source analysis of the late human auditory evoked potentials. J Cogn Neurosci 1989; 1:326-355.

20. Picton TW, Van Roon P, Armilio ML, Berg P, Ille N, Scherg M et al. Intracerebral sources of human auditory-evoked potentials. Audiol Neurootol 1999; 4:64-79.

21. Alain C, Woods DL and Knight RT. A distributed cortical network for auditory sensory memory in humans. Brain Res 1998; 812:23-37.

22. Rupp A et al. Fast temporal interactions in human auditory cortex. Neuroreport 2000; 11:3731-3736.

23. Rupp A, Gutschalk A, Hack S and Scherg M. Temporal resolution of the human primary auditory cortex in gap detection. Neuroreport 2002; 13:2203-2207.

Acknowledgements: This work was supported by grants from the Canadian Institutes of Health Research (ClHR), and the Natural Sciences and Engineering Research Council of Canada. The first author was supported, in part, by a ClHR Training Grant, and by the International Council for Canadian Studies. We wish to thank Chenghua Wang, James Qi, and He Yu for technical support. 